\title{
Estratégias de cuidado com a pele do recém-nascido em unidade de internação neonatal
}

\author{
Newborn skin care strategies in neonatal unit \\ Estrategias de cuidado de la piel del recién nacido en unidad neonatal
}

Bruna Schiphorst Delgado ${ }^{1}$, Roberta Costa ${ }^{1}$, Stéfany Nayara Petry Dal Vesco', Flávia Aguiar Santos ${ }^{1}$, Simone Vidal Santos ${ }^{1,2 *}$

ORCID IDS

Delgado BS (DD https://orcid.org/0000-0002-8412-7118 Costa R (D) https://orcid.org/0000-0001-6816-2047 Vesco SNP (D) https://orcid.org/0000-0001-9713-247X Santos FA (iD) https://orcid.org/0000-0002-9729-0299 Santos SV (D) https://orcid.org/0000-0002-5086-6326

\section{COMO CITAR}

Delgado BS, Costa R, Vesco SNP, Santos FA, Santos SV. Estratégias de cuidado com a pele do recém-nascido em unidade de internação neonatal. ESTIMA, Braz. J. Enterostomal Ther., 16: e1319. https://doi.org/10.30886/estima.v16.745_PT

\begin{abstract}
RESUMO
Objetivo: Identificar as estratégias de cuidado com a pele dos recém-nascidos (RNs) utilizadas nos centros de referência do Método Canguru (MC) do Brasil. Métodos: Estudo exploratório descritivo, de abordagem qualitativa, realizado com 13 enfermeiras atuantes nos diferentes centros de referência do MC no Brasil. Os dados foram coletados por meio de um formulário online. A análise foi feita de forma descritiva, comparando-se as diferentes realidades. Resultados: Elaboraram-se quatro categorias para o cuidado da pele do RN advindas da experiência dos participantes: estratégias adotadas pela equipe relacionadas aos cuidados com a pele do RN; manejo da equipe de saúde em relação ao banho e troca de fralda; cuidados com a fixação de dispositivos em RNs; e estratégias adotadas pela equipe no RN sob fototerapia. Conclusão: As enfermeiras mostraram-se capacitadas e aptas a atuar em seus campos de experiência e vivência. As respostas indicaram que existe preocupação em evitar a utilização de utensílios e materiais nocivos à pele do RN.
\end{abstract}

DESCRITORES: Recém-nascido; Unidades de terapia intensiva neonatal; Pele; Método Canguru; Enfermagem neonatal; Estomaterapia.

1 Universidade Federal de Santa Catarina - Departamento de Enfermagem - Florianópolis/SC - Brasil.

¿Universidade Federal de Santa Catarina - Hospital Universitário Professor Polydoro Ernani de São Thiago - Florianópolis/SC - Brasil. Autor correspondente: simonevidal75@gmail.com

Recebido: 4 Apr 2019 | Aceito: 10 Jul 2019 


\section{ABSTRACT}

Objective: To identify skin care strategies for newborns (NB) used in reference centers of the Kangaroo-Mother Care Method (KMC) in Brazil. Methods: Descriptive exploratory study, using a qualitative approach, performed with 13 nurses working in the different reference centers of the KMC in Brazil. Data were collected through an online form. Analysis was performed descriptively, comparing the different studies realities. Results: Four categories were described regarding skin care of NB through the experience of the nurses: strategies adopted by the team regarding skin care of NBs; dealing with the health team regarding bath and dipper disposal; care regarding fixation of devices in NBs, and strategies adopted by the NB team under phototherapy. Conclusion: The nurses presented capacity, and they were highly trained to act in their areas of experience and knowledge. The answers indicated that there is a great concern in avoiding the usage of materials and devices that could cause harm to NB skin.

KEYWORDS: Infant, Newborn; Neonatal Intensive Care Units; Skin; Kangaroo-Mother Care Method; Neonatal Nursing. Stomatherapy.

\section{RESUMEN}

Objetivo: identificar las estrategias de cuidado de la piel del recién nacido (RN) utilizadas en los centros de referencia del Método Canguro (MC) en Brasil. Métodos: Estudio exploratorio descriptivo con enfoque cualitativo, realizado con 13 enfermeras que trabajan en los diferentes centros de referencia de MC en Brasil. Los datos fueron recolectados a través de un formulario online. A análise foi feita de forma descritiva, comparando-se as diferentes realidades. Resultados: se elaboraron cuatro categorías para el cuidado de la piel del RN a partir de la experiencia de los participantes: estrategias adoptadas por el equipo relacionadas con el cuidado de la piel del RN; gestión del equipo de salud en relación con el baño y el cambio de pañales; cuidado con la fijación de dispositivos en RN; y estrategias adoptadas por el equipo en el recién nacido bajo fototerapia. Conclusión: Las enfermeras estaban calificadas y podían trabajar en sus campos de experiencia. As respostas indicaram que existe preocupação em evitar a utilização de utensílios e materiais nocivos à pele do RN. Las respuestas indicaron que existe la preocupación de evitar el uso de utensilios y materiales dañinos para la piel del RN.

DESCRIPTORES: Recién nacido; Unidades de cuidados intensivos neonatales; Piel; Método canguro; Enfermería Neonatal; Estomaterapia.

\section{INTRODUÇÃO}

Estima-se que, anualmente, nasçam aproximadamente 20 milhões de bebês pré-termos e de baixo peso no mundo, sendo que um terço desses morre antes mesmo de completar um ano de vida ${ }^{1}$. Mais de $60 \%$ dos partos prematuros ocorrem em países subdesenvolvidos e o Brasil está entre os 10 países com maiores índices de nascimentos antes do tempo, assim como estão também os Estados Unidos, a Índia e a Nigéria, constatando que esse é um problema global ${ }^{2}$. Em 2015, observou-se que aproximadamente 303 mil mulheres morreram por causas relacionadas à gravidez, 2,7 milhões de bebês morreram durante os primeiros 28 dias de vida e 2,6 milhões de bebês nasceram natimortos ${ }^{3}$.

Dada a magnitude do problema, a Organização Mundial de Saúde (OMS) idealiza um mundo no qual todas as mulheres e recém-nascidos $(\mathrm{RNs})$ possam receber cuidados de qualidade em todo o desenvolver da gravidez, do parto e do período pós-natal. Os cuidados pré-natais (CPNs) compõem uma plataforma de estratégias com vistas à promoção da saúde, ao rastreio, ao diagnóstico e à prevenção de doenças. A qualidade de serviços prestados durante a gravidez e o parto pode evitar muitas mortes que vêm ocorrendo ${ }^{1,3}$.
A fim de colaborar para a qualidade do cuidado e diminuir a mortalidade, o Sistema Único de Saúde (SUS) disponibiliza uma norma de atenção humanizada não somente ao RN pré-termo (RNPT), mas a toda a família, e faz dela coparticipante dos cuidados para passar por esse processo de forma tranquila e confiante ${ }^{1,3}$.

Os avanços da medicina e da tecnologia têm possibilitado que a grande maioria dos RNPT e de baixo peso consiga se desenvolver e crescer com saúde. Em contrapartida, esses bebês que nascem prematuramente são considerados frágeis, vulneráveis e necessitam de cuidados especiais para sobreviver. Várias ações têm sido estimuladas para melhorar o padrão de atendimento a essa população, e dentre essas destaca-se, no Brasil, a política governamental de Atenção Humanizada ao RN de Baixo Peso - Método Canguru (MC) ${ }^{1}$.

Inicialmente, o MC foi idealizado no Instituto Materno Infantil de Bogotá, Colômbia, sendo o ato de colocar o RN em contato pele a pele com a mãe uma alternativa para reduzir os custos da assistência, melhorar os cuidados prestados aos RNPT e promover o vínculo precoce entre mãe e bebê $\hat{e}^{4}$. No Brasil, o MC vem sendo inserido em algumas unidades desde a década de 1990 e, em 2000, o Ministério da Saúde (MS) lançou a Portaria no 693 de 5 de julho, atualizada em 
2007 pela Portaria no 1.683 , que estabelece os parâmetros necessários para implantação do método nas unidades e também para habilitar a equipe multiprofissional $1^{5}$.

O MC é dividido em três etapas e se inicia no prénatal, quando é diagnosticada a gestação de alto risco para o parto prematuro. A primeira etapa ocorre na Unidade de Terapia Intensiva Neonatal (UTIN) ou na Unidade de Cuidados Intermediários Neonatal Convencional (UCINCo), quando o bebê nasce e ainda está instável e depende de diversos cuidados da equipe. Nessa etapa, os pais devem ser encorajados pela equipe de saúde a participar dos cuidados do filho e podem iniciar a participação na hora do banho e na troca de fralda ${ }^{1}$.

A segunda etapa depende da disponibilidade da mãe em permanecer com o $\mathrm{RN}$ no hospital e, na maior parte do tempo, na posição canguru. Essa etapa ocorre na Unidade de Cuidados Intermediários Neonatal Canguru (UCINCa), uma ala diferenciada da UTIN na qual a mãe é a principal cuidadora do $\mathrm{RN}^{1}$.

A terceira ocorre a partir da alta hospitalar, que se inicia quando o RN alcança o peso mínimo de 1.600 gramas, encontra-se clinicamente estável e apresenta ganho ponderal progressivo. A mãe precisa estar motivada para levar o $\mathrm{RN}$ para casa e deve assumir o compromisso de realizar a posição canguru pelo maior tempo possível. As consultas são realizadas de forma ambulatorial pela equipe multiprofissional, sendo a primeira 48 horas após a alta e, pelo menos, uma vez por semana nas demais. O RN recebe alta da terceira etapa quando completa 2.500 gramas $^{1}$.

Para disseminação do $\mathrm{MC}$, foram criados centros de referência nacionais e estaduais, com a formação de tutores, para propiciar a implantação do método em diversos hospitais do país. Atualmente, no Brasil, existem cinco centros de referência nacional e 27 de referência estadual, sendo essas unidades consideradas locais para o desenvolvimento de boas práticas com o $\mathrm{RN}^{4}$. Dentre as boas práticas, destacam-se os cuidados com a pele do RN.

A pele é o maior órgão do corpo humano, composta pela epiderme e derme, duas camadas que se encontram firmemente aderidas e estão apoiadas acima do tecido subcutâneo. A epiderme é constituída por quatro camadas, sendo a camada mais externa o estrato córneo, que exerce a função de barreira contra a perda transepidérmica de fluídos, invasão de agentes do meio externo e auxilia na proteção da pele. A derme é formada por fibras de colágeno e elastina que garantem a sustentação, a elasticidade e a resistência da pele ${ }^{6}$.
As práticas de cuidado com a pele do $\mathrm{RN}$ estão presentes desde o nascimento e geram preocupações constantes aos profissionais que atuam em unidade neonatal ${ }^{7,8}$. A pele do $\mathrm{RN}$, principalmente do RNPT, tem características anatômicas e fisiológicas específicas, devido à sua imaturidade; é mais fina, delicada, sensível e torna essa população mais propensa e susceptível ao desenvolvimento de lesões e de infecção ${ }^{6,9}$. Por esse motivo, o planejamento da assistência de enfermagem e a implementação de ações que visem à avaliação e cuidados com a pele são essenciais para a qualidade da assistência e para o sucesso do tratamento em neonatologia ${ }^{10}$.

Os centros de referência vêm se tornando locais de desenvolvimento de boas práticas assistenciais. Nesse cenário, o cuidado com a pele do RN necessita de atenção redobrada do enfermeiro e de sua equipe. Estima-se que essas unidades neonatais constituem campos férteis nos quais possam surgir novas tecnologias que auxiliem nos cuidados com a pele do RN.

Diante desse contexto, elaborou-se a seguinte questão de pesquisa: quais estratégias de cuidado com a pele do RN são utilizadas nos centros de referência do Método Canguru do Brasil? O estudo tem como objetivo identificar as estratégias de cuidado com a pele dos $\mathrm{RN}$ nos centros de referência do MC do Brasil.

\section{MÉTODO}

Trata-se de estudo exploratório descritivo, de abordagem qualitativa, realizado a partir do macroprojeto de pesquisa intitulado "Tecnologias de cuidado ao recém-nascido e sua família no MC: contribuições dos enfermeiros de UTIN", desenvolvido na Universidade Federal de Santa Catarina. Esse recorte buscou identificar as estratégias de cuidado com a pele do $\mathrm{RN}$ utilizadas pelos enfermeiros nos centros de referência do MC do Brasil.

Todos os centros de referência foram convidados à participação no estudo, no entanto, obtiveram-se a carta de anuência de 27 . O contato ocorreu por intermédio do coordenador dos centros. Foram convidados 55 enfermeiros, por meio de carta-convite enviada por endereço eletrônico. Desses, 27 não retornaram ao $e$-mail, 15 aceitaram participar mas não preencheram o formulário e 13 enfermeiros, de centros de referência diferentes, participaram efetivamente da pesquisa. Os critérios de inclusão foram atuar há pelo menos seis meses na unidade e ser tutor ou consultor do 
MC. Delimitou-se, como critério de exclusão, o afastamento do trabalho nos últimos seis meses que antecediam a coleta de dados, seja por licença maternidade ou doença.

A pesquisa foi realizada nas unidades neonatais de 13 centros de referência do MC no Brasil localizados em Santa Catarina, São Paulo, Rio de Janeiro, Paraná, Ceará, Maranhão, Pernambuco, Piauí, Amapá, Pará, Espírito Santo e Tocantins. Os dados foram obtidos por meio da aplicação de um formulário online elaborado no programa Google Docs® e respondido pelas enfermeiras atuantes nessas unidades no período de junho a dezembro de 2016.

O formulário de coleta de dados foi composto por 94 questões, variando entre múltipla escolha e perguntas abertas, que possibilitavam às enfermeiras discorrer sobre o tema. Esse foi dividido em três sessões contendo: caracterização dos participantes; dados sobre a instituição; e questões sobre as tecnologias de cuidados na assistência ao RN. Este estudo focou em perguntas referentes à terceira sessão do formulário, voltadas ao cuidado da pele do RN na UTIN, UCINCo e UCINCa.

O processo de análise de dados foi realizado por meio de agrupamentos de similaridades de conteúdos que permitiu identificar as estratégias utilizadas pelas enfermeiras no cuidado com a pele do $\mathrm{RN}$ em diversos aspectos da prática assistencial.

Foram respeitados os preceitos éticos da Resolução no 466/2012 do Conselho Nacional de Saúde ${ }^{11}$ e a pesquisa foi aprovada pelo Comitê de Ética em Pesquisa da Universidade Federal de Santa Catarina sob o parecer CAAE 44889615.6.3003.5328. Os participantes assinaram o Termo de Consentimento Livre e Esclarecido.

\section{RESULTADOS}

Participaram da pesquisa 13 enfermeiras que atuam nos Centro de Referência do MC no Brasil, todas do sexo feminino, com idade variando entre 20 a 60 anos e tempo de formação entre 11 e 20 anos. Dessas, nove são tutoras e quatro são consultoras do MC no Brasil.

A partir das respostas dos formulários, elaboraram-se quatro categorias, voltadas ao cuidado da pele do RN, referentes à experiência dos enfermeiros de 13 centros de referência do MC no Brasil, quais sejam: Estratégias adotadas pela equipe de enfermagem relacionadas aos cuidados com a pele do RN; Realização do banho e troca de fralda; Cuidados com a fixação de dispositivos em RN; e Estratégias adotadas com o RN sob fototerapia.

\section{Estratégias adotadas pela equipe de enfermagem relacionadas aos cuidados com a pele do RN}

Verificaram-se condutas semelhantes e o surgimento de protocolos específicos da unidade. Evidenciaram-se a preocupação em evitar o uso de adesivos diretamente sobre a pele, assim como o uso de produtos para proteção.

"Evitar uso de substância, especialmente abrasivas, diminuir o uso de fitas adesivas, cuidado na retirada de adesivos, evitar exposições e manuseios excessivos.” (E1)

"Incubadora umidificada para RN menores de 1,000 gramas, retirada dos adesivos com umidificação.” (E4)

"Rotina escrita para cuidados com a pele do prematuro e comissão de prevenção de lesões de pele." (E6)

"Protocolo de lesão de pele; cuidados com oxímetros."(E10)

"Cavilom ${ }^{\circledR}$ barreira; Dersani ${ }^{\circledR}$ age; banho a cada 72 horas (bebês de muito baixo peso).”(E11)

"Uso de óleos. Não fixar fitas adesivas direto da pele. Usar fitas adesivas hipoalergênicas. Usar o tipo de antisséptico adequado para o peso do RN.” (E12)

"Creme de ureia, placas de hidrocoloides, mudança de decúbito, massagem de conforto.” (E13)

Constatou-se a diversidade de condutas, entretanto surgiram intervenções semelhantes quanto aos cuidados com a pele do RN. As enfermeiras descreveram que procuram evitar o uso de fitas adesivas, pois podem se tornar nocivas à pele. Frequentemente, respostas como uso de óleo, creme e placas de hidrocoloide são as melhores condutas adotadas. Embora não existam recomendações na literatura sobre a utilização de creme de ureia em neonatos, o uso desse produto foi relatado por uma das entrevistadas.

\section{Realização do banho e troca de fralda}

Com relação ao banho do $\mathrm{RN}$, observou-se que o protocolo e a rotina de cada unidade variaram, pois foram citados, por alguns participantes, o banho do RN e por outros apenas a higienização do períneo. No que se refere 
à troca de fralda, foi descrito que é realizada lateralmente, a fim de evitar complicações.

"Banho somente quando necessário, ou quando o bebê se apresenta estável, respeitando os sinais e tolerância, utilizando a técnica de enrolamento e a troca de fraldas com o cuidado de não elevar os MMII, sempre convidando a mãe a participar.” (E1)

"Segue-se protocolo cuidados com a pele; banho conforme peso e IG; troca fraldas lateralizada." (E3)

"De acordo com o recomendado pelo Método Canguru, ratificado por um protocolo." (E5)

"Banho humanizado, ofurô e troca de fralda lateralizada."(E6)

"Não são realizados banhos em recém-nascidos prematuros, apenas higiene do períneo.” (E8)

"De acordo com nosso protocolo de lesão de pele e seguindo os procedimentos operacionais padrão: banho cada 48 horas, troca de fraldas, usa algodão e água morna nada perfumado.”(E10)

"O banho é feito em dias alternados e dependendo a gravidade do recém-nascido, a troca de fralda e feita de 3 em 3 horas, antes da dieta ou sempre que necessário."(E12)

O banho nos $\mathrm{RN}$ apareceu como estratégia especifica de cada unidade, não sendo algo engessado, pois cada unidade aparenta ter sua rotina interna com relação à higienização corporal do bebê. O banho também foi descrito como uma maneira de implementar outras ações de cuidado, como a integração da enfermagem e família nesse momento.

\section{Cuidados com a fixação de dispositivos em RN}

Nesse item, identificou-se, novamente, a realidade de cada unidade, surgindo respostas que mostram a disponibilidade ou não de materiais necessários para a prática da enfermagem no cuidado ao RN.

"Curativos menos agressivos à pele, esparadrapo o mínimo possível.” (E2)
“Minimiza-se uso de adesividades, uso de hidrocoloide."(E3)

"Fixação de sonda gástrica tipo gatinho, fixação de TOT e CPAP com Tensoplast, retirada dos adesivos com uso de Dersani.”(E4)

"Curativo transparente que consiga ficar por sete dias e que não seja irritante para a pele. O TOT é fixado com Tensoplast ${ }^{\circledR}$, sonda orogástrica é fixada com fio de gaze no osso zigomático.”(E5)

“Abocath e PICC são fixados com filme transparente.”(E6)

"Utilizamos micropore e priorizamos o menor tamanho na aderência da pele.” (E7)

"Evitando excesso de fixações utilizando materiais apropriados para cada cateter. Utilizamos curativo de hidrocoloide extrafino e bandagem elástica para envolver sensores."(E10)

“TOT, CPAP, cateter de oxigênio, sonda gástrica são fixados com esparadrapo, mas antes colocamos Duoderme. PICC e Abocath são fixados com curativo filme estéril, quando tem disponível no hospital, sendo a troca feita a cada sete dias, desde que limpo e íntegro o curativo, ou então com gazes e micropore, sendo trocado a cada 48 horas." (E12)

Identificaram-se a utilização de diferentes materiais com foco no baixo potencial de lesão na pele, bem como estratégias diferenciadas no momento de fixação dos dispositivos. As respostas dos participantes evidenciaram que o cuidado prestado ao $\mathrm{RN}$ apresenta especificidades que variam conforme a disponibilidade de materiais e rotina de cada serviço.

\section{Estratégias adotadas com o RN sob fototerapia}

"Proteção dos olhos, exposição do máximo de área luz. Evitar uso de substâncias na pele. Uso de fraldas.”(E1)

“Óculos de proteção, alternar com posição canguru, temperatura axilar e controle da diurese." (E2)

"Posturamento, rolinhos, sucção não nutritiva (prescrição de chupeta se necessário).”(E3) 
“Troca da máscara a cada 24 horas e cada 3 horas desligada a fototerapia e retirada por alguns minutos máscara.” (E5)

"Uso de óculos protetor e se possível aleitamento materno."

“Proteção do papel filme para reduzir o frio[...].” (E9)

"Nenhum tipo de creme na pele, mudança de decúbito, controle de temperatura axilar de 4 em 4 horas." (E10)

"Proteção ocular com óculos de tecidos (UCINCa) e proteção ocular com óculos descartáveis (UTIN).” (E11)

"Controle de temperatura, de diurese, uso de proteção ocular." (E12)

As enfermeiras, em sua grande maioria, descreveram preocupações com os olhos do RN no momento da fototerapia. Evidenciaram-se, também, ações voltadas ao cuidado com a pele, tais como evitar o uso de substâncias tópicas que possam ser nocivas e a necessidade de reposicionamento no leito.

\section{DISCUSSÃO}

As principais estratégias adotadas demonstraram que as enfermeiras têm conhecimentos sobre os cuidados necessários e imprescindíveis para prevenção de lesões na pele do RN. Um tópico importante apontado pela maioria dos participantes foi a necessidade de evitar o uso de adesivos. As lesões de pele relacionadas a adesivos médicos (medical adhesive related skin injury - MARSI) ocorrem quando a ligação entre o adesivo e a pele é maior do que a coesão entre as camadas da pele e incluem lesão mecânica, lesão por tensão ou formação de bolhas e rasgões da pele. $\mathrm{Na}$ população neonatal, o mecanismo mais frequente observado de MARSI é a decapagem epidérmica ${ }^{12}$.

O uso de adesivos para fixação de dispositivos como tubo orotraqueal (TOT), cateteres nasogástricos, punções venosas, entre outros, pode acarretar perda epidérmica considerável do RN. A fim de evitar essas lesões, deve-se proteger a pele utilizando um produto que propicie barreira entre a pele e o adesivo, como a placa de hidrocoloide ${ }^{12}$. Esse fato é comprovado e utilizado pelos profissionais desses centros de referência quando surgem respostas positivas ao uso da placa de hidrocoloide como uma maneira de evitar as lesões. Somado a essa conduta, deve-se atentar para o cuidado na remoção das fixações, utilizando gaze embebida em solução fisiológica ou óleo mineral, a fim de prevenir lesões durante esse procedimento ${ }^{12}$.

Os cuidados prestados na unidade neonatal abrangem a realização de diversas técnicas e cuidados, como o uso de soluções antissépticas, procedimentos invasivos, manutenção da temperatura, posicionamento do RN, higiene corporal, fixação/remoção de adesivos, uso de dispositivos invasivos e monitores, entre outros procedimentos e ações que predispõem os RN ao surgimento de lesões ${ }^{13,14}$.

Estudos apontaram que as principais causas dessas lesões de pele estão relacionadas ao uso e à fixação de dispositivos, fontes de calor, uso de drogas por via intravenosa, incontinência e uso de produtos químicos ${ }^{(13-15)}$.

O uso de antissépticos, por exemplo, é considerado um dilema no cuidado com a pele do $\mathrm{RN}$, pois, ao mesmo tempo em que auxiliam na prevenção de infecções, também podem causar lesão. Outro ponto a ser considerado é a inexistência de um produto adequado para uso seguro nesses pacientes. Deve-se atentar para a formulação desses produtos, evitando soluções que contenham álcool, priorizando soluções aquosas e irrigando o local com solução salina após a desinfecção, a fim de retirar o excesso de antisséptico, evitar absorção, irritação química e formação de lesões ${ }^{16}$.

$\mathrm{O}$ continuous positive airway pressure (CPAP), sistema para ventilação não invasiva que fornece pressão contínua de ar nas vias aéreas superiores, é considerado um dos maiores causadores de lesão nos RN. O dispositivo consiste no uso de traqueias e de pronga ou máscara nasal adaptada ao nariz da criança. As traqueias são fixadas na cabeça do $\mathrm{RN}$, na região temporal bilateralmente ou na região frontal, dependendo do fabricante. A pronga fica em contato com a columela nasal, asa das narinas e supralabial e a máscara com toda a região ao redor do nariz. A pressão constante exercida pelo contato das traqueias, da pronga ou da máscara nasal com a pele pode causar lesão por pressão. Uma revisão sistemática concluiu que os RNPT com idade gestacional (IG) menor que 30 semanas são mais suscetíveis a esse tipo de lesão e apontou como solução o uso de barreiras, como a placa de hidrocoloide nas áreas de contato do CPAP com a pele ${ }^{17}$.

Com relação ao banho, encontraram-se divergências nas respostas das participantes deste estudo. As unidades têm suas rotinas e ações preconizadas, as quais são seguidas pelos profissionais a fim de manter a homeostase do RN. O banho é considerado um procedimento rotineiro que segue uma 
tradição cultural, tem como objetivo a remoção de resíduos e da colonização bacteriana, porém altera o pH cutâneo e interfere no manto ácido bacteriano, comprometendo a integridade da pele ${ }^{8}$.

Faz-se também necessário o cuidado no momento de troca das fraldas, uma vez que as dermatites de fralda são lesões que ocorrem pelo contato da pele com urina e fezes, o que faz com que a ureia seja convertida em amônia, tornando o $\mathrm{pH}$ da região mais alcalino. Acredita-se que o uso de antibióticos pode favorecer o surgimento de dermatites de fralda, pois esses medicamentos alteram a consistência das fezes. Para prevenir sua ocorrência, a troca das fraldas deve ser realizada sempre que houver sujidade, utilizando-se pano macio ou algodão e água para limpeza da região perianal do neonato, pois o uso de produtos químicos pode causar irritação na pele, principalmente nos prematuros extremos ${ }^{7,18}$. Outro cuidado importante na troca de fralda é o uso de produtos que formem uma barreira protetora na pele, a fim de evitar dermatites causadas pelo contato da fralda com a pele; essa afecção é muito comum e tem prevalência de 7 a $50 \%$ dos neonatos ${ }^{19}$.

É fundamental que, além do conhecimento sobre as particularidades anatômicas e fisiológicas da pele dos neonatos, os enfermeiros identifiquem os riscos para surgimento de lesões, com vistas à melhoria da qualidade da assistência e, consequentemente, tornando-a mais humanizada, reduzindo, assim, as complicações decorrentes das lesões, o tempo de hospitalização, a mortalidade, os custos para o tratamento, além de minimizar o sofrimento físico e emocional dos RN em condições críticas e de seus familiares ${ }^{20}$.

$\mathrm{Na}$ unidade neonatal, procedimentos como banho, hidratação com óleos emolientes, uso de soluções para antissepsia, fixação de adesivos e prevenção da perda de água e calor são cuidados a serem monitorados com frequência, assim como os procedimentos que envolvam troca de curativos, inserção de cateteres, punções capilares, arteriais e venosas, mudanças de decúbito, entre outros. Todos esses cuidados são prestados na assistência de enfermagem e, se mal executados, podem levar ao surgimento de lesões, devido à frequência dos procedimentos no processo de hospitalização que geralmente é longo ${ }^{21}$.

Outro procedimento que pode acarretar danos na pele do RN é a fototerapia, cuja finalidade é o tratamento da hiperbilirrubinemia por meio de exposição do RN à luz artificial gerada por aparelhos. É um método não invasivo e de alto impacto na diminuição dos níveis de bilirrubina plasmática, já que a exposição à luz auxilia na quebra da bilirrubina, facilitando sua metabolização e excreção ${ }^{22}$. Para que a fototerapia seja efetiva, a equipe de enfermagem deve estar atenta a alguns aspectos, como a superfície corporal exposta à luz, distância apropriada entre o RN e a fonte luminosa, irradiância do aparelho de fototerapia, proteção ocular, controle dos sinais vitais, avaliação do balanço hídrico e evitar o uso de substâncias na pele do $\mathrm{RN}^{23}$.

A proteção ocular é necessária a fim de evitar danos na retina do bebê e, para tal, deve-se priorizar protetores oculares que não necessitam de adesivos para fixação direta na pele, para evitar lesões. Hoje, já existem, no mercado, protetores oculares que se ajustam à cabeça do $\mathrm{RN}$ de forma adequada, por meio de velcro ou elástico, que devem ser utilizados com cautela para que não fiquem apertados e ocasionem lesões de pele nem largos, deixando os olhos desprotegidos. É importante, também, retirar os óculos ao desligar a fonte luminosa para avaliar os olhos quanto à drenagem, edema $\mathrm{e}$ evidência de infecção, fornecer estimulação visual e encorajar a interação entre pais e bebês, conforme apropriado, com base no estado clínico da criança ${ }^{22}$.

A avaliação da pele do RN é uma estratégia de cuidado para prevenir lesões e deve ser realizada pelo enfermeiro por meio de instrumentos de avaliação, como a Escala de Condição da Pele do RN (ECPRN), que avalia secura, eritema e lesão ${ }^{24}$. $\mathrm{O}$ intervalo de avaliação pode variar entre seis a 24 horas e deve se basear no escore de condição da pele, bem como na avaliação dos riscos, como IG, tratamento com fototerapia, uso de dispositivos, edema, uso de drogas sedativas, diarreia, entre outros fatores que podem contribuir para o surgimento de lesões.

\section{CONCLUSÃO}

Este estudo demonstra a importância de um enfermeiro bem preparado e ciente das peculiaridades que envolvem a assistência ao neonato, principalmente no que se refere à manutenção da integridade da pele. Esse cuidado precisa ser observado, avaliado e executado minuciosamente e efetivamente para prevenir danos que podem ser devidamente evitados.

Diante dos resultados, percebe-se que as enfermeiras se mostram capacitadas e aptas a atuar em seus campos de experiência e vivência. Indicam, ainda, que existe preocupação em adotar as melhores estratégias de cuidado, a fim de evitar a utilização de materiais e produtos nocivos que possam comprometer a integridade da pele do RN. 
Apesar de serem centros de referência distintos, distribuídos nas variadas regiões do Brasil, as respostas dos participantes se mostraram homogêneas quanto às estratégias de cuidado com a pele, bem como às rotinas específicas de cada instituição quanto ao manejo da assistência.

Este estudo contribui para evidenciar um tema pouco abordado na literatura e fomentar discussões acerca das melhores práticas de cuidado, fundamentais para a qualidade do trabalho, proteção ao desenvolvimento e segurança da população neonatal.

Como limitações do estudo, aponta-se a baixa adesão dos enfermeiros dos centros de referência na devolução do formulário preenchido.

Recomenda-se que a equipe de enfermagem esteja em constante busca de atualização e capacitação para atuar efetivamente no cuidado da pele do RN, fomentar boas práticas, prevenir complicações e contribuir para a segurança do paciente neonatal.

\section{FINANCIAMENTO}

Esta pesquisa foi realizada com financiamento do Conselho Nacional de Desenvolvimento Científico e Tecnológico (CNPq). Edital Universal - Chamada 14/2013.

\section{CONTRIBUIÇÃO DOS AUTORES}

Conceitualização, Delgado BS; Costa R; Vesco SNP; Santos FA e Santos SV; Metodologia, Degado BS e Costa R; Investigação, Delgado BS e Costa R; Redação - Primeira versão, Delgado BS; Costa R; Vesco SNP; Santos FA e Santos SV; Redação - Revisão \& Edição, Delgado BS; Costa R; Vesco SNP; Santos FA e Santos SV; Aquisição de Financiamento, Costa R; Recursos, Delgado BS e Costa R; Supervisão, Costa R.

\section{REFERÊNCIAS}

1. Ministério da Saúde (BR). Atenção humanizada ao recémnascido de baixo peso: Método Canguru: manual técnico (3a ed.). Brasília: MS; 2017.

2. World Health Organization. Born too soon: the global action report on preterm birth [Internet]. Genebra: WHO; 2012 [citado 23 maio 2017]. Disponível em: http://www.who.int/ pmnch/media/news/2012/201204_borntoosoon-report.pdf

3. World Health Organization. WHO recommendations on antenatal care for a positive pregnancy experience [Internet]. Genebra: WHO; 2016 [citado 23 maio 2017]. Disponível em: http://apps.who.int/iris/bitstre am/10665/250796/1/9789241549912-eng.pdf?ua=1

4. Sanches MTC, Costa R, Azevedo VMGO, Morsch DS, Lamy ZC, organizadores. Método Canguru no Brasil: 15 anos de política pública. São Paulo: Instituto de Saúde; 2015.

5. Ministério da Saúde (BR). Atenção humanizada ao recémnascido: Método Canguru: caderno do tutor. Brasília: MS; 2014.

6. Visscher MO, Adam R, Brink S, Odio M. Newborn infant skin: physiology, development, and care. Clin Dermatol. 2015;33(3):271-80. https://doi.org/10.1016/j. clindermatol.2014.12.003

7. Santos SV, Costa R. Prevention of newborn skin lesions: knowledge of the nursing team. Texto Contexto Enferm 2015;24(3):731-9. https://doi.org/10.1590/0104-07072015 011230014

8. Lund C. Bathing and beyond: current bathing controversies for newborn infants. Adv Neonatal Care 2016;16(Suppl 5):13-20. https://doi.org/10.1097/ANC.0000000000000336
9. Cheever KH, Hinkle JL. Brunner \& Suddarth: tratado de enfermagem médico-cirúrgica. 13a ed. Vol. 1. Rio de Janeiro: Guanabara Koogan; 2015.

10. Santos SV, Costa R. Treatment of skin lesions in newborn children: meeting the needs of nursing staff. Rev Esc Enferm USP. 2014;48(6):985-92. https://doi.org/10.1590/S0080623420140000700004

11. Conselho Nacional de Saúde (BR). Resolução n. 466, de 12 de dezembro de 2012 [Internet]. Brasília; 2012 [citado 23 maio 2017]. Disponível em: http://bvsms.saude.gov.br/bvs/ saudelegis/cns/2013/res0466_12_12_2012.html

12. Lund $\mathrm{CH}$. Medical adhesives in the NICU. Newborn Infant Nurs Rev. 2014;14(4):160-5. https://doi.org/10.1053/j. nainr.2014.10.001

13. Visscher $M$, Narendran $V$. Neonatal infant skin: development, structureand function. Newborn Infant Nurs Rev. 2014;14(4):13541. https://doi.org/10.1053/j.nainr.2014.10.004

14. Nist MD, Rodgers EA, Ruth BM, Bertoni CB, Bartman T, Keller $L A$, et al. Skin rounds: a quality improvement approach to enhance skin care in the neonatal intensive care unit. Adv Neonatal Care. 2016;16(Suppl 5):33-41. https://doi. org/10.1097/ANC.0000000000000337

15. Cousins Y. Wound care considerations in neonates. Nurs Stand. 2014;28(46);61-70. https://doi.org/10.7748/ ns.28.46.61.e8402

16. Sathiyamurthy S, Banerjee J, Godambe SV. Antiseptic use in the neonatal intensive care unit - a dilemma in clinical practice: an evidence-based review. World J Clin Pediatr 2016;5(2):159-71. https://doi.org/10.5409/wjcp.v5.i2.159 
17. Imbulana DI, Manley BJ, Dawson JA, Davis PG, Owen LS. Nasal injury in preterm infants receiving non-invasive respiratory support: a systematic review. Arch Dis Child Fetal Neonatal Ed. 2018;103:29-35. https://doi.org/10.1136/ archdischild-2017-313418

18. Biranjia-Hurdoyal SD, Pandamikum L. A study to investigate the prevalence of nappy rash among babies aged 0 to 36 months old in a tropical country. Austin J Dermatol. 2015;2(2):id1040.

19. Šikić PM, Maver U, Marčun VN, Mičetić-Turk D. Diagnosis and management of diaper dermatitis in infants with emphasis on skin microbiota in the diaper area. Int J Dermatol. 2018;57(3):265-75. https://doi.org/10.1111/ ijd.13748

20. Faria TF, Kamada I. Lesões de pele em neonatos em cuidados intensivos neonatais. Enfermería Global. 2018;49:220-8. https://doi.org/10.6018/eglobal.17.1.273671
21. Johnson DE. Extremely preterm infant skin care: a transformation of practice aimed to prevent harm. Adv Neonatal Care. 2016;16(Suppl 5):26-32. https://doi. org/10.1097/ANC.0000000000000335

22. Yurdakök M. Phototherapy in the newborn: what's new? J Pediatr Neonat Individual Med. 2015;4(2):e040255. https:// doi.org/10.7363/040255

23. Abdelazeem KS, Soliman AA, Askar EAA. Efficacy of intensive phototherapy in management of neonatal hyperbilirubinemia in neonatal unit of Assiut university children hospital. J Neonatal Biol. 2017;6(3):1000266. https://doi.org/10.4172/2167-0897.1000266

24. Schardosim JM, Ruschel LM, Motta GCP, Cunha MLC. Cross-cultural adaptation and clinical validation of the Neonatal Skin Condition Score to Brazilian Portuguese. Rev Latino-Am Enfermagem. 2014;22(5):834-41. https://doi. org/10.1590/0104-1169.3456.2487 